\title{
Elementary Uncertain Renewal Reward Theorem and Its Strict Proof
}

\author{
Xiaojing Shi, Xingfang Zhang* \\ School of Mathematical Sciences, Liaocheng University, Liaocheng, China \\ Email: shixiaojing2014@126.com, "Zhangxingfang2005@126.com
}

Received 27 January 2016; accepted 12 February 2016; published 16 February 2016

Copyright (C) 2016 by authors and OALib.

This work is licensed under the Creative Commons Attribution International License (CC BY). http://creativecommons.org/licenses/by/4.0/

(C) (i) Open Access

\begin{abstract}
Uncertain renewal reward process, of which the interarrival times and rewards (or costs) are regarded as uncertain variables, is an important branch of Liu's uncertainty theory. At present, there is a lack of strict proof for the elementary theorem. Therefore, the paper gives its strict proof with two lemmas by some techniques.
\end{abstract}

Keywords

Elementary Theorem, Renewal Reward Process, Uncertain Process, Uncertainty Theory

Subject Areas: Mathematical Analysis

\section{Introduction}

In probability theory, renewal process and renewal reward process are two important uncertain processes in which interarrival times and rewards are regarded as random variables.

Note that probability theory is applicable only when the obtained probability is close enough to the real frequency. Otherwise, some counterintuitive results will happen [1]. But in real life, we are often lack of observed data or historical data to estimate the probability distributions of interarrival times and reward, so we have to invite some domain experts to evaluate their belief degree of the interarrival times and reward. Since human tends to overweight unlikely events (Kahneman and Tversky [2]), the belief degree may have a much larger than the real frequency. Thus probability theory fails to model the renewal process and renewal reward process in this situation. In order to resolve these problems, an uncertainty theory is founded by Liu [3] and refined by Liu [4] based on normality, duality, subadditivity and product axioms. Nowadays, uncertainty theory has been applied to uncertain programming [5] [6], uncertain process [7]-[10] etc. [11] [12], uncertainty theory. In the framework of uncertainty theory, Liu [13] first assumed the interarrival times and reward of an renewal process as uncertain variables, and proposed an uncertain renewal process. Then Liu [4] also proposed an

${ }^{*}$ Corresponding author. 
uncertain renewal reward process which interarrival times and rewards were both regarded as uncertain variables and gave the an elementary renewal reward theorem. At present, there is a lack of strict proof for the elementary theorem. Therefore, the paper will give its strict proof with two lemmas by some techniques.

\section{Preliminary}

Definition 1. (Liu [3]) Let $\mathcal{L}$ be a $\sigma$-algebra on nonempty set $\Gamma$. A set function $\mathcal{M}$ is called an uncertain measure if it satisfies the following axioms:

Axiom 1. (Normality) $M\{\Gamma\}=1$; for the universal set $\Gamma$;

Axiom 2. (Duality) $\mathcal{M}\{\Lambda\}+\mathcal{M}\left\{\Lambda^{c}\right\}=1$ for any event $\Lambda$;

Axiom 3. ( Subadditivity) For every countable sequence of events $\left\{\Lambda_{i}\right\}$, we have

$$
\mathcal{M}\left\{\bigcup_{i=1}^{\infty} \Lambda_{i}\right\} \leq \sum_{i=1}^{\infty} \mathcal{M}\left\{\Lambda_{i}\right\}
$$

In this case, the triple $(\{\Lambda\}, \mathcal{L}, \mathcal{M})$ is called an uncertainty space.

In [14], Liu further presented the following axiom:

Axiom 4. (Product Axiom) Let $\left(\{\Lambda\}_{k}, \mathcal{L}_{k}, \mathcal{M}_{k}\right)$ be uncertainty spaces for $k=1,2, \cdots$. Then the product uncertain measure $\mathcal{M}$ is an uncertain measure satisfying

$$
\mathcal{M}\left\{\prod_{i=1}^{n} \Lambda_{k}\right\}=\min _{1 \leq k \leq n} \mathcal{M}_{k}\left\{\Lambda_{k}\right\}
$$

where $\Lambda_{k}$ are arbitrarily chosen events from $\mathcal{L}_{k}$ for $k=1,2, \cdots$, respectively.

Definition 2. (Liu [3]) An uncertain variable is a measurable function $\xi$ from an uncertainty space $(\Gamma, \mathcal{L}, \mathcal{M})$ to the set of real numbers, i.e., for any Borel set $B$ of real numbers, the set $\{\xi \in B\}=\{\gamma \in \Gamma \mid \xi(\gamma) \in B\}$ is an event.

Definition 3. (Liu [3]) The uncertainty distribution $\Phi$ of an uncertain variable $\xi$ is defined by $\Phi(x)=\mathcal{M}\{\xi \leq x\}$ for any real number $x$.

Definition 4. (Liu [4]) An uncertainty distribution $\Phi$ is said to be regular if its inverse function $\Phi^{-1}(\alpha)$ exists and is unique for each $\alpha \in(0,1)$.

Definition 5. (Liu [14]) The uncertain variables $\xi_{1}, \xi_{2}, \cdots, \xi_{m}$ are said to be independent if

$$
\mathcal{M}\left\{\bigcap_{i=1}^{m}\left(\xi_{i} \in B_{i}\right)\right\}=\min _{1 \leq i \leq m} \mathcal{M}\left\{\xi_{i} \in B_{i}\right\}
$$

for any Borel sets $B_{1}, B_{2}, \cdots, B_{m}$ of real numbers.

Definition 6. (Liu [3] (2007)) The expected value of uncertain variable $\xi$ is defined by

$$
E[\xi]=\int_{0}^{\infty} \mathcal{M}\{\xi \geq r\} \mathrm{d} r-\int_{-\infty}^{0} \mathcal{M}\{\xi \leq r\} \mathrm{d} r
$$

provided that at least one of the two integrals is finite.

Theorem 1. (Liu [4]) Let $\xi$ be an uncertain variable with uncertainty distribution $\Phi$. If the expected value exists, then $E[\xi]=\int_{0}^{1} \Phi^{-1}(\alpha) \mathrm{d} \alpha$.

Theorem 2. (Liu [14]) Let $\xi_{1}, \xi_{2}, \cdots, \xi_{n}$ be independent uncertain variables with uncertainty distributions $\Phi_{1}, \Phi_{2}, \cdots, \Phi_{n}$, respectively. If $f\left(x_{1}, x_{2}, \cdots, x_{n}\right)$ is strictly increasing with respect to $x_{1}, x_{2}, \cdots, x_{m}$ and strictly decreasing with respect to $x_{m+1}, x_{2}, \cdots, x_{n}$, then $\xi=f\left(\xi_{1}, \xi_{2}, \cdots, \xi_{n}\right)$ is an uncertain variable with uncertainty distribution

$$
\Psi(x)=\sup _{f\left(x_{1}, x_{2}, \cdots, x_{n}\right)=x}\left(\min _{1 \leq i \leq m} \Phi_{i}\left(x_{i}\right) \wedge \min _{m+1 \leq i \leq n}\left(1-\Phi_{i}\left(x_{i}\right)\right)\right)
$$


and inverse uncertainty distribution

$$
\Psi^{-1}(\alpha)=f\left(\Phi_{1}^{-1}(\alpha), \Phi_{2}^{-1}(\alpha), \cdots, \Phi_{n}^{-1}(\alpha)\right) .
$$

In particular, if $\xi_{1}, \xi_{2}, \cdots, \xi_{n}$ have a common uncertainty distribution $\Phi$, then $\xi=\xi_{1}+\xi_{2}+\cdots+\xi_{n}$ have a uncertainty distribution $\Psi(x)=\Phi\left(\frac{x}{n}\right)$.

Definition 7. (Liu [3]) Let $\xi, \xi_{1}, \xi_{2}, \cdots$ be a sequence of uncertain variables with uncertainty distributions $\Phi, \Phi_{1}, \Phi_{2}, \cdots$, respectively, then $\xi_{i}$ is said to converge in distribution to $\xi$ if $\lim \Phi_{i}(x)=\Phi(x)$ at every continuous point $x$ of $\Phi$.

\section{Uncertain Renewal Reward Process}

Definition 8. (Liu [13]) Let $T$ be a index set and let $(\Gamma, \mathcal{L}, \mathcal{M})$ be an uncertainty space. An uncertain process is a measurable function from $T \times(\Gamma, \mathcal{L}, \mathcal{M})$ to the set of real numbers, i.e., for any $t \in T$ and any Borel set $B$ of real numbers, the set $\left\{X_{t} \in B\right\}=\left\{\gamma \in \Gamma \mid X_{t}(\gamma) \in B\right\}$ is an event.

Definition 9. (Liu [13]) Let $\xi_{1}, \xi_{2}, \cdots$ be independent and identical distribution(iid) positive uncertain variables. Define $S_{0}=0$ and $S_{n}=\xi_{1}+\xi_{2}+\cdots+\xi_{n}$ for $n \geq 1$. Then the uncertain process $N_{t}=\max _{n \geq 0}\left\{n \mid S_{n} \leq t\right\}$ is called a renewal process.

Note that event $\left\{N_{t} \leq k\right\}$ is same with event $\left\{S_{K+1} \leq t\right\}$.

For an uncertain renewal process, Liu [4] proved that $\frac{N_{t}}{t}$ converges in mean to $\frac{1}{\xi_{1}}$, i.e.,

$$
\lim _{t \rightarrow \infty} \frac{E\left[N_{t}\right]}{t}=E\left[\frac{1}{\xi_{1}}\right] .
$$

Definition 10. (Liu [4]) Let $\xi_{1}, \xi_{2}, \cdots$ be iid uncertain interarrival times, and let $\eta_{1}, \eta_{2}, \cdots$ be uncertain rewards. It is also assume that $\xi_{1}, \eta_{1}, \xi_{2}, \eta_{2}, \cdots$ are independent. Then

$$
R_{t}=\sum_{i=1}^{N_{t}} \eta_{i}
$$

is called a renewal reward process, where $N_{t}$ is the renewal process.

Theorem 3. (Liu [4]) Let $R_{t}$ be a renewal reward process with uncertain interarrival times $\xi_{1}, \xi_{2}, \cdots$ and uncertain rewards $\eta_{1}, \eta_{2}, \cdots$. If those interarrival times $\xi_{1}, \xi_{2}, \xi_{3} \cdots$ and rewards $\eta_{1}, \eta_{2}, \eta_{3}, \cdots$ have uncertainty distributions $\Phi$ and $\Psi$, then $R_{t}$ has an uncertainty distribution

$$
\Upsilon_{t}(x)=\max _{k \geq 0}\left(1-\Phi\left(\frac{t}{k+1}\right)\right) \wedge \Psi\left(\frac{x}{k}\right) .
$$

Here we set $x / k=\infty$ and $\Phi(x / k)=1$ when $k=0$.

Liu gave an elementary uncertain renewal reward theorem in the book [4] (see latter Theorem 4). But, it is not strict to proof of the theorem. Therefore, in the following we strict prove it by two lemmas.

Lemma 1. If $\Phi(x)$ and $\Psi(x)$ are nonnegative continuous strict increasing functions on $[0,+\infty)$, and $\Phi(0)=\Psi(0)=0, \lim _{x \rightarrow+\infty} \Phi(x)=\lim _{x \rightarrow+\infty} \Psi(x)=1$, then

(i) for given $x \in[0,+\infty)$, there exists $s_{x} \in(0,+\propto)$ such that

$$
\begin{aligned}
\sup _{s \geq 0}((1-\Phi(s)) \wedge \Psi(s x)) & =\max _{s \geq 0}((1-\Phi(s)) \wedge \Psi(s x)) \\
& =\left(1-\Phi\left(s_{x}\right)\right) \wedge \Psi\left(s_{x} x\right)=1-\Phi\left(s_{x}\right)=\Psi\left(s_{x} X\right)
\end{aligned}
$$

(ii) 


$$
\lim _{t \rightarrow+\infty} \max _{k \geq 1}\left(1-\Phi\left(\frac{t}{k+1}\right)\right) \wedge \Psi\left(\frac{t x}{k}\right)=\sup _{s \geq 0}(1-\Phi(s)) \wedge \Psi(s x)
$$

Proof. Proof of (i) is easy. In following we prove (ii). Note that we have the following facts:

$$
\begin{gathered}
\frac{t}{k+1} \leq \frac{t}{k} \leq s_{x},\left(1-\Phi\left(\frac{t}{k+1}\right)\right) \wedge \Psi\left(\frac{t}{k} x\right)=\Psi\left(\frac{t}{k} x\right) \leq \Psi\left(s_{x} x\right), \\
s_{x} \leq \frac{t}{k+1} \leq \frac{t}{k},\left(1-\Phi\left(\frac{t}{k+1}\right)\right) \wedge \Psi\left(\frac{t}{k} x\right)=\left(1-\Phi\left(\frac{t}{k+1}\right)\right) \leq \Psi\left(s_{x} x\right), \\
\frac{t}{k+1} \leq s_{x} \leq \frac{t}{k},\left(1-\Phi\left(\frac{t}{k+1}\right)\right) \wedge \Psi\left(\frac{t}{k} x\right) \geq \Psi\left(s_{x} x\right) .
\end{gathered}
$$

For given $t \in\left[s_{x}+1,+\infty\right)$, there exists $k_{0}=\left[\frac{t}{s_{x}}\right]$ such that

$$
\frac{\frac{t}{s_{x}}}{\left[\frac{t}{s_{x}}\right]+1} s_{x} \leq s_{x} \leq \frac{\frac{t}{s_{x}}}{\left[\frac{t}{s_{x}}\right]} s_{x}
$$

and for any integer $k \neq_{0} k$ such that

$$
\frac{t}{k+1}<\frac{t}{\left[\frac{t}{s_{x}}\right]+1}<s_{x}<\frac{t}{\left[\frac{t}{s_{x}}\right]}<\frac{t}{k}
$$

Thus, when $t>s_{x}+1$,

$$
\max _{k \geq 1}\left(1-\Phi\left(\frac{t}{k+1}\right)\right) \wedge \Psi\left(\frac{t x}{k}\right)=\left(1-\Phi\left(\frac{\frac{t}{s_{x}}}{\left[\frac{t}{s_{x}}\right]+1} s_{x}\right) \wedge \Psi\left(\frac{\frac{t}{s_{x}} x}{\left[\frac{t}{s_{x}}\right]}\right) .\right.
$$

Also,

$$
\lim _{t \rightarrow+\infty} \frac{\frac{t}{s_{x}}}{\left[\frac{t}{s_{x}}\right]+1}=\lim _{t \rightarrow+\infty} \frac{\frac{t}{s_{x}}}{\left[\frac{t}{s_{x}}\right]}=1
$$

and function $\Psi$ at $s_{x}$ is continuous, thus

$$
\lim _{t \rightarrow+\infty} \max _{k \geq 1}\left(1-\Phi\left(\frac{t}{k+1}\right)\right) \wedge \Psi\left(\frac{t x}{k}\right)=\lim _{t \rightarrow+\infty}\left(1-\Phi\left(\frac{\frac{t}{s_{x}}}{\left[\frac{t}{s_{x}}\right]+1} s_{x}\right) \wedge \Psi\left(\frac{\frac{t}{s_{x}}}{\left[\frac{t}{s_{x}}\right]} s_{x} x\right)=\Psi\left(s_{x} x\right) .\right.
$$

i.e.,

$$
\lim _{t \rightarrow+\infty} \max _{k \geq 1}\left(1-\Phi\left(\frac{t}{k+1}\right)\right) \wedge \Psi\left(\frac{t x}{k}\right)=\Psi\left(s_{x} x\right)=\sup _{s \geq 0}(1-\Phi(s)) \wedge \Psi(s x) .
$$

Lemma 2. If conditions of Lemma 1 are satisfied, and 


$$
\int_{0}^{+\infty}\left(1-\sup _{s \geq 0}(1-\Phi(s)) \wedge \Psi(s x)\right) \mathrm{d} x
$$

converge, then

$$
\int_{0}^{+\infty}\left(1-\max _{k \geq 1}\left(1-\Phi\left(\frac{t}{k+1}\right)\right) \wedge\left(\frac{t x}{k}\right)\right) \mathrm{d} x
$$

consistent convergent on $[0,+\infty)$ about $t$.

Proof. It follows from process of proof of Lemma 1 that, for any $t \in[0,+\infty)$,

$$
\sup _{s \geq 0}(1-\Phi(s)) \wedge(s x) \leq \max _{k \geq 1}\left(1-\Phi\left(\frac{t}{k+1}\right)\right) \wedge \Psi\left(\frac{t x}{k}\right) .
$$

Therefore, for any $t \in[0,+\infty)$,

$$
1-\max _{k \geq 1}\left(1-\Phi\left(\frac{t}{k+1}\right)\right) \wedge \Psi\left(\frac{t x}{k}\right) \leq 1-\sup _{s \geq 0}(1-\Phi(s)) \wedge \Psi(s x) .
$$

also,

$$
\int_{0}^{+\infty}\left(1-\sup _{s \geq 0}(1-\Phi(s)) \wedge \Psi(s x)\right) \mathrm{d} x
$$

is convergent, then

$$
\int_{0}^{+\infty}\left(1-\max _{k \geq 1}\left(1-\Phi\left(\frac{t}{k+1}\right)\right) \wedge \Psi\left(\frac{t x}{k}\right)\right) \mathrm{d} x
$$

is consistent convergent on $[0,+\infty)$ about $t$.

Theorem 4. (Elementary uncertain renewal reward theorem, Liu [4]) Let $R_{t}$ be a renewal reward process with uncertain interarrival times $\xi_{1}, \xi_{2}, \cdots$ and uncertain rewards $\eta_{1}, \eta_{2}, \cdots$ If $E\left[\eta_{1} / \xi_{1}\right]$ exists, then $\lim _{t \rightarrow \infty} \frac{E\left[R_{t}\right]}{t}=E\left[\frac{\eta_{1}}{\xi_{1}}\right]$. If those interarrival times $\xi_{1}, \xi_{2}, \cdots$ and rewards $\eta_{1}, \eta_{2}, \cdots$ have regular uncertainty distribution $\Phi$ and $\Psi$ satisfy the following conditions $\Phi(0)=\Psi(0)=0$, and $\lim _{x \rightarrow+\infty} \Phi(x)=\lim _{x \rightarrow+\infty} \Psi(x)=1$, then

$$
\lim _{t \rightarrow \infty} \frac{E\left[R_{t}\right]}{t}=\int_{0}^{1} \frac{\Psi^{-1}(\alpha)}{\Phi^{-1}(1-\alpha)} \mathrm{d} \alpha .
$$

Proof. Firstly, note that uncertainty distribution of $\eta_{1} / \xi_{1}$ is

$$
\gamma_{\eta_{1} / \xi_{1}}(x)=\sup _{s \geq 0}(1-\Phi(s)) \wedge \Psi(s x),
$$

and

$$
E\left[\eta_{1} / \xi_{1}\right]=\int_{0}^{+\infty}\left(1-\sup _{s \geq 0}((1-\Phi(s)) \wedge \Psi(s x))\right) \mathrm{d} x=\int_{0}^{1} \frac{\Psi^{-1}(\alpha)}{1-\Phi^{-1}(\alpha)} \mathrm{d} \alpha .
$$

Since the uncertainty distribution of $R_{t}$ is

$$
G_{t}(x)=\max _{k \geq 0}\left(1-\Phi\left(\frac{t}{k+1}\right)\right) \wedge \Psi\left(\frac{x}{k}\right),
$$

and the uncertainty distribution of $R_{t} / t$ is

$$
G_{t}(x)=\max _{k \geq 0}\left(1-\Phi\left(\frac{t}{k+1}\right)\right) \wedge \Psi\left(\frac{t x}{k}\right),
$$


using Lemma 2 we have

$$
\begin{aligned}
\lim _{t \rightarrow+\infty} \frac{E\left[R_{t}\right]}{t} & =\lim _{t \rightarrow+\infty} \int_{0}^{+\infty}\left(1-\left(1-\Phi\left(\frac{t}{k+1}\right)\right) \wedge \Psi\left(\frac{t}{k} x\right)\right) \mathrm{d} x \\
& =\int_{0}^{+\infty}\left(1-\sup _{s \geq 0}(1-\Phi(s)) \wedge \Psi(s x)\right) \mathrm{d} x=\int_{0}^{1} \frac{\Psi^{-1}(\alpha)}{1-\Phi^{-1}(\alpha)} \mathrm{d} \alpha=E\left[\frac{\eta_{1}}{\xi_{1}}\right]
\end{aligned}
$$

\section{Conclusion}

This paper provides a strict proof of elementary uncertain renewal reward theorem by some technics.

\section{Acknowledgements}

This work was supported by National Natural Science Foundation of China Grants No. 61273044 and No. 11471152.

\section{References}

[1] Liu, B.D. (2012) Why Is the Reaneed for Uncertainty Theory. Journal of Uncertain Systems, 6, 3-10.

[2] Kahneman, D. and Tversky, A. (1979) Prospect Theory: An Analysis of Decision Underrisk. Econometrica, 47, 263292. http://dx.doi.org/10.2307/1914185

[3] Liu, B.D. (007) Uncertainty Theory. 2nd Edition, Springer-Verlag, Berlin. http://dx.doi.org/10.1007/978-3-540-73165-8_5

[4] Liu, B.D. (2010) Uncertainty Theory: A Branch of Mathematics for Modeling Human Uncertainty. Springer-Verlag, Berlin.

[5] Gao, Y. (2011) Shortest Path Problem with Arc Lengths. Computers and Mathematics with Applications, 62, 25912600. http://dx.doi.org/10.1016/j.camwa.2011.07.058

[6] Gao, Y. (2012) Uncertain Models for Single Facility Location Problem on Networks. Applied Mathematical Modelling, 36, 2592-2599. http://dx.doi.org/10.1016/j.apm.2011.09.042

[7] Yao, K. and Li, X. (2012) Uncertain Alternating Renewal Process and Its Application. IEEE Transactions on Fuzzy Systems, 20, 1154-1160. http://dx.doi.org/10.1109/TFUZZ.2012.2194152

[8] Yao, K. (2012) Uncertain Calculus with Renewal Process. Fuzzy Optimization and Decision Making, 11, $285-297$. http://dx.doi.org/10.1007/s10700-012-9132-y

[9] Zhang, X., Ning, Y. and Meng, G. (2013) Delayed Renewal Process with Uncertain Interarrival Times. Fuzzy Optimization and Decision Making, 12, 79-87. http://dx.doi.org/10.1007/s10700-012-9144-7

[10] Chen, X.W. (2012) Variation Analysis of Uncertain Stationary Independent Increment Process. European Journal of Operational Research, 222, 312-316. http://dx.doi.org/10.1016/j.ejor.2012.05.010

[11] Zhang, X. and Li. X. (2014) A Semantic Study of the First-Order Predicate Logic with Uncertainty Involved. Fuzzy Optimization and Decision Making, 13, 357-367. http://dx.doi.org/10.1007/s10700-014-9184-2

[12] Chen, X.W., Kar, S. and Ralescu, D.A. (2012) Cross-Entropy Measure of Uncertain Variables. Information Sciences, 201, 53-60. http://dx.doi.org/10.1016/j.ins.2012.02.049

[13] Liu, B.D. (2008) Fuzzy Process, Hybrid Process and Uncertain Process. Journal of Uncertain Systems, 2, 3-16.

[14] Liu, B.D. (2009) Some Research Problems in Uncertainty Theory. Journal of Uncertain Systems, 3, 3-10. 